\title{
Ruin probabilities in models with a Markov chain dependence structure
}

\author{
C. Constantinescu ${ }^{a}$, D. Kortschak ${ }^{a}$, V. Maume-Deschamps ${ }^{b}$ \\ ${ }^{a}$ Department of Actuarial Science, Faculty HEC, University of Lausanne, \\ Extranef Building, CH-1015 Lausanne, Switzerland. \\ ${ }^{b}$ Université de Lyon, Université Lyon 1, Institut de Science Financière et d'Assurances, \\ 50 Avenue Tony Garnier, F-69007 Lyon, France.
}

\begin{abstract}
In this paper we derive explicit expressions for the probability of ruin in a renewal risk model with dependence described-by/incorporated-in the real-valued random variable $Z_{k}=-c \tau_{k}+X_{k}$, namely the loss between the $(k-1)$-th and the $k$-th claim. Here $c$ represents the constant premium rate, $\tau_{k}$ the inter-arrival time between the $(k-1)$-th and the $k$-th claim and $X_{k}$ is the size of the $k$-th claim. The dependence structure among $\left(Z_{k}\right)_{k>0}$ is given/driven by a Markov chain with a transition kernel satisfying an ordinary differential equation with constant coefficients.
\end{abstract}

Keywords: Ruin probability, dependence, Markov chain, random walk, rational Laplace transform, density satisfying ODE with constant coefficients.

\section{Introduction}

Since the classical discrete time risk model (seen as a random walk) due to de Finetti (1957), the ruin probability is an established attempt to evaluate the solvency of an insurance company. In this context, we are interested in providing explicit expressions for the probability of ruin.

While most classical models assume independence between inter-arrival times and claim amounts, we consider a special dependence between the positive random variable $\tau_{k}$ representing the $k$-th inter-arrival time and the subsequent claim size denoted by the positive random variable $X_{k}$. More specifically, we consider a random walk like structure of the real-valued random variable

$$
Z_{k}=X_{k}-c \tau_{k}, \quad k=1,2, \ldots
$$

where $c$ represents the premium rate and $\mathbb{E}\{Z\}<0$. The sum of increments (random walk if independent increments)

$$
W_{n}=\sum_{k=1}^{n} Z_{k}
$$

Email address: corina.constantinescu@unil.ch, dominik.kortschak, veronique.maume@univ-lyon1.fr (C. Constantinescu ${ }^{a}$, D. Kortschak ${ }^{a}$, V. Maume-Deschamps ${ }^{b}$ ) 
represents the loss of the company after $n$ claims. For an initial capital amount $u>0$, the company is considered to be insolvent if $W_{n}>u$, and the probability $\psi(u)$ of this event is called the ruin probability. W.l.o.g., in this paper $\mathbb{P}(Z=0)=0$.

By characterizing the dependence in renewal risk models via the series of identical distributed losses $\left(Z_{k}\right)_{k>0}$, one has two possible scenarios:

S1. $Z_{1}, Z_{2}, \ldots$ are independent (random walk structure)

S2. $Z_{1}, Z_{2}, \ldots$ are not independent (with multiple possible dependence structures among $Z_{k} \mathrm{~s}$ ).

Since in risk theory literature several cases preserving $\left(Z_{k}\right)_{k>0}$ independent (scenario \$1) have already been addressed (see Albrecher and Teugels (2006), Ambagaspitiva (2009) or Badescu et al. (2009)), we will concentrate on a case pertaining to the second scenario. For a recent and more exhaustive survey of other dependence structures in risk theory see Asmussen and Albrecher (2010) [Chapter XIII].

The challenging part of choosing $\left(Z_{k}\right)_{k>0}$ to be dependent is the impossibility of relying on standard random walk theory. However, we show that one can still use the decomposition into positive-negative parts

$$
Z=I Z^{+}+(1-I) Z^{-}, \quad I \sim \operatorname{Bernoulli}(p),
$$

with $Z^{+}=\{Z \mid Z>0\}$ and $Z^{-}=\{Z \mid Z<0\}$, introduced in the random walk treatment, to find closed form solutions for the probability of ruin $\psi(u)$. Similarly to Albrecher et al. (2010), we derive the exact forms via solving ordinary differential equations. The difference is that while in Albrecher et al. (2010) one exploits the independence of, and between $\tau_{k}$ and $X_{k}$, here one focuses on the dependence structure of the losses $Z_{k}$, whereas the pair $\tau_{k}, X_{k}$ can have any dependent structure (even independence). Thus the necessary conditions to be imposed for calculating exactly the ruin probability are no longer on the densities of $\tau$ and $X$ as in Albrecher et al. (2010), but on densities pertinent to $Z$ or $Z^{+} / Z^{-}$.

More specifically, in this paper we consider a dependence structure for the dependent losses $\left(Z_{k}\right)_{k>0}$ given by a Markov chain with a transition kernel $p_{k}(x, y)$ (starting at $x$, jumps from state $x$ to state $y$ at time $k$ with probability $p_{k}$ ) that can be written as a product of two functions on each quadrant. The condition needed is that combinations of these functions further satisfy certain ODEs with constant coefficients (see Assumption 1). This condition is not intuitively evident. However, besides the mathematical amenability, the motivation/interpretation of such a choice of dependent model is the following. The weight $p=\mathbb{P}(Z>0)$ not being constant over time, but dependent on the previous step in a deterministic way, influences the tendency of the future behavior of the steps $Z$. Thus, by the choice of the mechanism to determine the next $p$, we can generate models where if one period was bad (we earn less than we spend i.e. $Z>0$ ) it is more likely than in the independent case that the next period will also be bad. 
To justify further our Markov chain structure choice, we present as a particular/ limiting case of it the case of independent $\left(Z_{k}\right)_{k>0}$, with densities of $Z^{+}$and $Z^{-}$satisfying ODEs with constant coefficients. A further particular case of it is the case present in risk theory literature, of the density of $Z$ satisfying an ODE with constant coefficients, as in Albrecher and Teugels (2006); Ambagaspitiva (2009); Badescu et al. (2009).

Similarly to Albrecher et al. (2010), where one transforms an integral equation for the probability of ruin into an ordinary differential equation, here we transform a system of integral equations into a system of ordinary differential equations that one can solve explicitly. This way we show that even under this dependence scenario, the probability of ruin has still a rational Laplace transform.

Thus, the classes of distributions for which one can identify the probability of ruin as having a rational Laplace transform are the following:

A. In the dependent case: Markov chain structure on $Z_{k}$ with a kernel satisfying certain ODE conditions, as in Propositions 10 2 and [3.

B. In the independent case: the classical result in random-walk theory of $Z^{+}$ with a rational Laplace transform, as in Proposition 4

C. In the independent case: the classical case in ruin theory literature of $Z$ with rational moment generating function as in Proposition 5

Note that for the distribution class $\square$ one can always find a dependence structure where the marginal distributions of $\tau$ and $X$ have rational Laplace transform (see Lemma 1 in Appendix A). Equally natural once can identify a dependence structure with marginals (of $\tau$ and $X$ ) not having a rational Laplace transform. For instance, one can have $\tau=Y_{1}+Y_{3}$ and $X=Y_{2}+Y_{3}$, where $Y_{1}, Y_{2}$ and $Y_{3}$ are independent, $Y_{1}$ and $Y_{2}$ have a rational Laplace transform, but $Y_{3}$ has not. Actually, $Y_{3}$ can have any positive distribution function, even heavy-tailed.

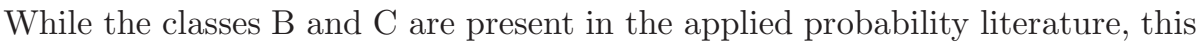
paper brings forth class $\$$ and shows that as in the other two classes of distributions, the probability of ruin can be derived by means of ODEs with constant coefficients.

The paper is organized as follows. In Section 2 we introduce a renewal risk model via a random walk with real-valued steps $Z_{k}$, and define the probability of ruin in this context. In Section 3, we analyze a risk model where the steps $Z_{k}$ are dependent via an order one Markov chain. We consider a Markov chain structure with a transition kernel expressed-through/described-by some ODE with constant coefficients and show that the ruin probability has a rational Laplace transform. In Section 4, we present the case of independent steps $Z_{k}$ as a particular, limiting case of the Markov chain dependence from Section 3. We reiterate/reformulate in our ODE language well-known results from random walk and risk literature. In Section 5, we look at a Markov chain dependent case with a transition kernel expressed through exponential densities, and for a numerical example we compare the values of probability of ruin under a Markov 
chain dependence structure with the independent increments one. After the conclusions from Section 6, in the Appendix we present some additional lemmas and some deferred proofs.

Note regarding notation and terminology. Depending on relevance and purpose, we will switch back and forth between the following equivalent concepts:

- the density function $f_{X}$ of the random variable $X$ satisfies an ODE with constant coefficients

- the moment generating function (mgf) $M_{X}(s)$ of the random variable $X$ is rational; or, equivalently, $X$ has a rational Laplace transform.

These are both equivalent to matrix exponentials (Bladt and Nielsen, 2010).

\section{Generic random variable $Z$}

Consider the collective risk model

$$
U(t)=u+c t-\sum_{k=1}^{N_{t}} X_{k},
$$

where $X_{k}$ is the $k$-th claim amount and $N_{t}$ is the number of claims that occurred up to time $t$. The ruin can occur only at the time of a claim. Immediately after the $k$-th claim, for $u \geq 0$, the reserve is

$$
U\left(T_{k}\right)=u+c T_{k}-\sum_{i=1}^{k} X_{i}=u+c \sum_{i=1}^{k} \tau_{i}-\sum_{i=1}^{k} X_{i}=u-\sum_{i=1}^{k} Z_{i} .
$$

The probability of ruin in infinite time is defined as

$$
\psi(u)=\mathbb{P}\left(\sup _{k \geq 1} \sum_{i=1}^{k} Z_{i}>u\right) .
$$

\section{3. $\left(Z_{k}\right)_{k \geq 1}$ dependent}

In this section we study the case where $\left(Z_{k}\right)_{k \geq 0}$ are dependent and assume to have an order one Markov chain structure. For a given $Z_{0}(=x)$, we define the probability of ruin by

$$
\psi(u, x)=\mathbb{P}\left(\sup _{k \geq 1} \sum_{i=1}^{k} Z_{i}>u \mid Z_{0}=x\right) .
$$

We denote the transition density of $Z$ by

$$
p(x, y) d x:=\mathbb{P}\left(Z_{i+1} \in d y \mid Z_{i}=x\right)=\mathbb{P}\left(Z_{i+1} \in d y \mid Z_{i}=x, Z_{i-1}=x, \ldots\right) .
$$


For $u \geq 0$, by conditioning on $Z_{1}$ one has

$$
\begin{aligned}
\psi(u, x) & =\mathbb{P}\left(Z_{1}>u \mid Z_{0}=x\right)+\mathbb{P}\left(Z_{1} \leq u, \sup _{k \geq 1} \sum_{i=2}^{k} Z_{i}>u-Z_{1} \mid Z_{0}=x\right) \\
& =\mathbb{P}\left(Z_{1}>u \mid Z_{0}=x\right)+\int_{-\infty}^{u} \mathbb{P}\left(\sup _{k \geq 1} \sum_{i=2}^{k} Z_{i}>u-Z_{1} \mid Z_{0}=x, Z_{1}=y\right) p(x, y) d y \\
& =\mathbb{P}\left(Z_{1}>u \mid Z_{0}=x\right)+\int_{-\infty}^{u} \mathbb{P}\left(\sup _{k \geq 1} \sum_{i=2}^{k} Z_{i}>u-Z_{1} \mid Z_{1}=y\right) p(x, y) d y \\
& =\int_{u}^{\infty} p(x, y) d y+\int_{-\infty}^{u} \psi(u-y, y) p(x, y) d y
\end{aligned}
$$

To obtain a system of ODEs for the ruin probability we will assume a rather special structure for the transition density, namely:

Assumption 1. Let

$$
p(x, y)= \begin{cases}g^{1}(x) h^{1}(y), & (x, y) \in I_{1} \\ g^{2}(x) h^{2}(y), & (x, y) \in I_{2} \\ g^{3}(x) h^{3}(y), & (x, y) \in I_{3} \\ g^{4}(x) h^{4}(y), & (x, y) \in I_{4}\end{cases}
$$

where $I_{k}$ denotes the $k$-th quadrant of the cartesian plane. Further let $g^{1}(x)>$ $0, g^{4}(x)>0$ for $x>0, g^{1}(x)=g^{4}(x)=0$ for $x<0, g^{1}(x), g^{4}(x)$ are linearly independent and $g^{1}(x)+g^{4}(x)=1$. Similarly $g^{2}(x)>0, g^{3}(x)>0$ for $x<$ $0, g^{2}(x)=g^{3}(x)=0$ for $x>0, g^{2}(x), g^{3}(x)$ are linearly independent and $g^{2}(x)+g^{3}(x)=1$. Further assume that for

$$
(k, m) \in\{(1,1),(1,2),(4,1),(4,2),(2,3),(2,4),(3,3),(3,4)\}
$$

there exists polynomials $q^{k, m}(x):=\sum_{i=0}^{n} q_{i}^{k, m} x^{i}$ with

$$
q^{k, m}\left(\frac{d}{d u}\right) g^{k}(u) h^{m}(u)=0 \quad u \neq 0
$$

An interpretation of this kind of dependence is given in Section 5

Remark 1. One can easily extend the results of this paper to a transition density of the form

$$
p(x, y)= \begin{cases}\sum_{i=1}^{n_{1}} g_{i}^{1}(x) h_{i}^{1}(y), & (x, y) \in I_{1} \\ \sum_{i=1}^{n_{2}} g_{i}^{2}(x) h_{i}^{2}(y), & (x, y) \in I_{2} \\ \sum_{i=1}^{n_{3}} g_{i}^{3}(x) h_{i}^{3}(y), & (x, y) \in I_{3} \\ \sum_{i=1}^{n_{4}} g_{i}^{4}(x) h_{i}^{4}(y), & (x, y) \in I_{4}\end{cases}
$$

with the obvious extension of the conditions to $g_{i}^{1}(x)$ and $h_{i}^{1}(y)$. Nevertheless note that this generalization is straight-forward (one needs to replace all matrices by corresponding block matrices). Hence, for ease of notation we consider only the case $n=1$. 
In order to evaluate the ruin probability we first need to check that for a given set of parameters the process tends to $-\infty$. Therefore one needs to evaluate the invariant distribution $f_{Z}(x)$ of $p(x, y)$ (see Lemma 4 in the Appendix), although this is not needed further for the derivation of the ruin probability. To find explicit expressions for $\psi(u, x)$ a key observation is:

Proposition 1. If Assumption 1 is fulfilled, then the probability of ruin has the form

$$
\psi(u, x)= \begin{cases}g^{1}(x) \psi^{1}(u)+g^{4}(x) \psi^{4}(u), & x>0 \\ g^{2}(x) \psi^{2}(u)+g^{3}(x) \psi^{3}(u), & x<0\end{cases}
$$

where $\psi^{i}=\int_{u}^{\infty} h^{i}(y) d y+\int_{0}^{u} \psi(u-y, y) h^{i}(y) d y$, for $i=1,3$ and $\psi^{i}=\int_{-\infty}^{0} \psi(u-$ $y, y) h^{4}(y) d y$, for $i=2,4$. Further, for $i=1, \ldots, 4$,

$$
\lim _{u \rightarrow \infty} \psi^{i}(u)=0 \text {. }
$$

Remark 2. Note that for $x>0, \psi^{1}(u)$ is the probability of ruin given that the first step (jump) is positive and $\psi^{4}(u)$ is the ruin probability given that the first step is negative. Similarly, for $x<0, \psi^{2}(u)$ is the probability of ruin given that the first step is positive and $\psi^{3}(u)$ is the ruin probability given that the first step is negative.

Next we provide a system of ODEs for the functions $\psi^{i}$.

Proposition 2. If Assumption 1 is fulfilled, then $\psi^{i}(u), i=1, \ldots, 4$ are a solution of the system of ODEs

$$
A \tilde{\psi}=0
$$

where $\tilde{\psi}=\left(\psi^{1}, \psi^{2}, \psi^{3}, \psi^{4}\right)^{T}$,

$$
A=\left(\begin{array}{cccc}
\frac{\left(r^{1,1}-q^{1,1}\right) q^{4,1}}{g c d\left(q^{1,1} q^{4,1}\right)} & 0 & 0 & \frac{q^{1,1} r^{4,1}}{g c d\left(q^{1,1}, q^{4,1}\right)} \\
\frac{r^{1,2} q^{4,2}}{g c d\left(q^{1,2}, q^{4,2}\right)} & -\frac{q^{1,2} q^{4,2}}{g c d\left(q^{1,2},,^{4,2}\right)} & 0 & \frac{q^{1,2} r^{4,2}}{g c d\left(q^{1,2}, q^{4,2}\right)} \\
0 & \frac{r^{2,3} q^{3,3}}{\operatorname{gcd}\left(q^{2,3} q^{3,3}\right)} & \frac{\left(r^{3,3}-q^{3,3}\right) q^{2,3}}{\operatorname{gcd}\left(q^{2,3}, q^{3,3}\right)} & 0 \\
0 & \frac{r^{2,4} q^{3,4}}{\operatorname{gcd}\left(q^{2,4}, q^{3,4}\right)} & \frac{q^{2,4} r^{3,4}}{\operatorname{gcd}\left(q^{2,4}, q^{3,4}\right)} & -\frac{q^{2,4} q^{3,4}}{g c d\left(q^{2,4}, q^{3,4}\right)}
\end{array}\right),
$$

$\operatorname{gcd}(a, b)$ denotes the greatest common divisor of the polynomials $a$ and $b$ and $r^{k, m}$ is defined by

$$
M_{k, m}(-s):=M_{g^{k} h^{m}}(-s):=\int_{-\infty}^{\infty} e^{-s x} g^{k}(x) h^{m}(x) d x=\frac{r^{k, m}(s)}{q^{k, m}(s)} .
$$

Further if $h^{1}(x)=h^{2}(x)$ then $\psi^{1}(u)=\psi^{2}(u)$ and if $h^{3}(x)=h^{4}(x)$ then $\psi^{3}(u)=$ $\psi^{4}(u)$.

Proof. Substituting (8) into the last two lines of the matrix $A$ leads to

$$
\begin{aligned}
& g^{1}(x)\left(\int_{u}^{\infty} h^{1}(y) d y+\int_{0}^{u}\left(\psi^{1}(u-y) g^{1}(y)+\psi^{4}(u-y) g^{4}(y)\right) h^{1}(y) d y\right) \\
& \quad+g^{4}(x) \int_{-\infty}^{0}\left(\psi^{2}(u-y) g^{2}(y)+\psi^{3}(u-y) g^{3}(y)\right) h^{4}(y) d y \\
& =g^{1}(x) \psi^{1}(u)+g^{4}(x) \psi^{4}(u)
\end{aligned}
$$


Since $g^{1}(x)$ and $g^{4}(x)$ are linearly independent we get the equations

$$
\begin{cases}\psi^{1}(u)-\int_{0}^{u}\left(\psi^{1}(u-y) g^{1}(y)+\psi^{4}(u-y) g^{4}(y)\right) h^{1}(y) d y & =\int_{u}^{\infty} h^{1}(y) d y \\ \psi^{4}(u)-\int_{-\infty}^{0}\left(\psi^{2}(u-y) g^{2}(y)+\psi^{3}(u-y) g^{3}(y)\right) h^{4}(y) d y & =0\end{cases}
$$

Analogously, using the linear independence of $g^{2}(x)$ and $g^{3}(x)$ from the last two lines of equation (3) one has the system

$$
\begin{cases}\psi^{2}(u)-\int_{0}^{u}\left(\psi^{1}(u-y) g^{1}(y)+\psi^{4}(u-y) g^{4}(y)\right) h^{2}(y) d y & =\int_{u}^{\infty} h^{2}(y) d y \\ \psi^{3}(u)-\int_{-\infty}^{0}\left(\psi^{2}(u-y) g^{2}(y)+\psi^{3}(u-y) g^{3}(y)\right) h^{3}(y) d y & =0\end{cases}
$$

In order to write this system of equations in matrix form, we introduce the operators

$K_{+}^{l, m} \psi:=\int_{0}^{u} \psi(u-y) g^{l}(y) h^{m}(y) d y \quad$ and $\quad K_{-}^{l, m} \psi:=\int_{-\infty}^{0} \psi(u-y) g^{l}(y) h^{m}(y) d y$

(note that $g^{l}, h^{m}$ vanish either on the positive or negative half-line). Further, denoting by 1 the identity operator, the above system of equations can be written as

$$
\left(\begin{array}{cccc}
K_{+}^{1,1}-1 & 0 & 0 & K_{+}^{4,1} \\
K_{+}^{1,2} & -1 & 0 & K_{+}^{4,2} \\
0 & K_{2,3}^{2,3} & K_{-}^{3,3}-1 & 0 \\
0 & K_{-}^{2,4} & K_{-}^{3,4} & -1
\end{array}\right)\left(\begin{array}{c}
\psi^{1} \\
\psi^{2} \\
\psi^{3} \\
\psi^{4}
\end{array}\right)=\left(\begin{array}{c}
-\int_{u_{\infty}}^{\infty} h^{1}(y) d y \\
-\int_{u}^{\infty} h^{2}(y) d y \\
0 \\
0
\end{array}\right)
$$

If $h^{1}(x)=h^{2}(x)$ then $K_{+}^{1,1}=K_{+}^{1,2}$ and $K_{+}^{4,1}=K_{+}^{4,2}$, hence it follows by subtracting the second line from the first line that $\psi^{1}(u)=\psi^{2}(u)$. Similarly if $h^{3}(x)=h^{4}(x)$, then we get by subtracting the fourth line from the third line that $\psi^{3}(u)=\psi^{4}(u)$, Hence the second part of the proposition follows.

For the first part we want to reduce this system of IDEs to a system of ODEs that one can solve. It is enough to act on the integral system with a matrix of the form

$$
\left(\begin{array}{cccc}
\frac{q^{1,1} q^{4,1}}{\operatorname{gcd}\left(q^{1,1}, q^{4,1}\right)} & 0 & 0 & 0 \\
0 & \frac{q^{1,2} q^{4,2}}{g c d\left(q^{1,2}, q^{4,2}\right)} & 0 & 0 \\
0 & 0 & \frac{q^{2,3} q^{3,3}}{\operatorname{gcd}\left(q^{2,3}, q^{3,3}\right)} & 0 \\
0 & 0 & 0 & \frac{q^{2,4} q^{3,4}}{\operatorname{gcd}\left(q^{2,4}, q^{3,4}\right)}
\end{array}\right)
$$

formed by operators of the type $q^{k, m}\left(\frac{d}{d u}\right)$ that annihilate each $K_{ \pm}^{k, m}$. Applying this matrix to the right-hand side of the equation leads to a homogeneous system, since the left-hand side becomes zero after differentiation. More specifically, for $i=1,2$,

$$
\begin{aligned}
\int_{u}^{\infty} h^{i}(y) d y=\int_{u}^{\infty} g^{1}(y) h^{i}(y) d y+\int_{u}^{\infty} g^{4}(y) h^{i}(y) d y \\
=\int_{0}^{\infty} g^{1}(u+y) h^{i}(u+y) d y+\int_{0}^{\infty} g^{4}(u+y) h^{i}(u+y) d y .
\end{aligned}
$$


Each term in the sum is annihilated by the corresponding operator,

$$
q^{1, i}\left(\frac{d}{d u}\right) \int_{0}^{\infty} g^{1}(u+y) h^{i}(u+y) d y=0,
$$

respectively

$$
q^{4, i}\left(\frac{d}{d u}\right) \int_{0}^{\infty} g^{4}(u+y) h^{i}(u+y) d y=0 .
$$

Now the result follows from Lemma 2 and 3 in the Appendix.

From Proposition 2 we get that $\psi^{i}(u)$ is a solution of a system of linear ODEs. The following theorem gives the explicit form of $\psi$. Its proof is given in the Appendix.

Proposition 3. If Assumption 1 is fulfilled, then

$$
\psi^{i}(u)=\sum_{j=1}^{n} \sum_{k=0}^{m} c_{j, k}^{i} u^{k} e^{-\lambda_{j} u},
$$

where $-\lambda_{j}$ are either the negative roots of the polynomial

$$
\frac{q^{1,1} q^{1,2} q^{2,3} q^{2,4} q^{3,3} q^{3,4} q^{4,1} q^{4,2}}{\operatorname{gcd}\left(q^{1,1}, q^{4,1}\right) \operatorname{gcd}\left(q^{1,2}, q^{4,2}\right) \operatorname{gcd}\left(q^{2,3}, q^{3,3}\right) \operatorname{gcd}\left(q^{2,4}, q^{3,4}\right)}
$$

or the roots of

$$
\begin{aligned}
1= & -M_{1,1}(-s) M_{3,3}(-s)-M_{1,1}(-s)-M_{3,3}(-s) \\
& -M_{2,3}(-s) M_{3,4}(-s) M_{4,2}(-s)\left(M_{1,1}(-s)-1\right) \\
& +M_{1,2}(-s) M_{2,3}(-s) M_{3,4}(-s) M_{4,1}(-s) \\
& +M_{2,4}(-s) M_{4,2}\left(M_{1,1}(-s) M_{3,3}(-s)-M_{1,1}(-s)-M_{3,3}(-s)+1\right) \\
& -M_{1,2}(-s) M_{2,4}(-s) M_{4,1}(-s)\left(M_{3,3}(-s)-1\right),
\end{aligned}
$$

with $M_{k, m}$ are defined as in (9).

Note that for a fixed $j, c_{j, k}^{i}$ are linearly dependent. To get the $c_{j, k}^{i}$ we can plug $\psi^{i}(u)$ into Equation (10), which finally results in a linear system of equations for $c_{j, k}^{i}$. Further note that by setting $u=0$ in the first two lines of Equation (10) we get the conditions

$$
\psi^{1}(0)=\int_{0}^{\infty} h^{1}(y) d y=1 \quad \text { and } \quad \psi^{2}(0)=\int_{0}^{\infty} h^{1}(y) d y=1 .
$$

An intuitive meaning of these expressions is that given that we have a positive jump at zero, if $u=0$ then ruin is certain.

\section{The connection with $\left(Z_{k}\right)_{k \geq 1}$ independent}

If we set $h^{1}(x)=h^{2}(x)$ as the density of $Z^{+}$satisfying an ODE with constant coefficients (e.g. phase-type distributions), $h^{3}(x)=h^{4}(x)$ as the density of $Z^{-}$, $g^{1}(x)=g^{2}(x)=\mathbb{P}(Z>0)$ and $g^{3}(x)=g^{4}(x)=\mathbb{P}(Z<0)$, then our model corresponds to the independent $\left(Z_{k}\right)_{k \geq 1}$ case. However, note that in this case 
Assumptions 1 are not fullfilled, since $g^{1}(x)$ and $g^{4}(x)$ are now not independent. A way around this problem is to set

$$
g^{1}(x)=g^{2}(x)=\mathbb{P}(Z>0) e^{-\theta x} \quad \text { and } \quad g^{3}(x)=g^{4}(x)=1-g^{1}(x) .
$$

Thus, letting $\theta \rightarrow 0$ one recovers the independent case.

Moreover, taking $\theta \rightarrow 0$ in (10) and then applying the appropriate differential operator (to eliminate the integrals on the right-hand side), one obtains a new (simpler) system of ODEs

$$
\left(\begin{array}{cc}
p r_{+}-q_{+} & p r_{+} \\
(1-p) r_{-} & (1-p) r_{-}-q_{-}
\end{array}\right)\left(\begin{array}{l}
\psi^{1} \\
\psi^{4}
\end{array}\right)=\left(\begin{array}{l}
0 \\
0
\end{array}\right)
$$

where $p=\mathbb{P}(Z>0)$. Let $*$ stand for either + or - . Then, here $q_{*}$ is polynomial (as in characteristic equation) describing the ODE with constant coefficients that the densities of $Z^{*}$, whether $r_{*}$ is defined through the rational moment generating functions of $Z^{*}$,

$$
M_{Z^{*}}(-s)=\frac{r_{*}(s)}{q_{*}(s)}
$$

Standard ordinary differential equation theory says that $\psi^{1}(u), \psi^{4}(u)$ and implicitely $\psi(u)$, are a linear combination of exponential functions, where the exponents are solutions of the polynomial equations

$$
\begin{aligned}
\left(p r^{+}-q^{+}\right)\left((1-p) r^{-}-q^{-}\right)-p(1 & -p) r^{+} r^{-} \\
& =q^{+} q^{-}-(1-p) r^{-} q^{+}-p r^{+} q^{-}=0 .
\end{aligned}
$$

Equation (11) is equivalent to $M_{Z}(s)=1$, further equivalent to $\psi(u)$ having a rational Laplace transform, which coincides with the result of Asmussen (1992) [Corollary 5.1.] for $Z^{+}$phase-type distributed. In the following proposition, we (re)state a slight extension of this corollary (from phase-type to rational Laplace transform class). Using our notations, we make further connections with other existing results from the $\left(Z_{k}\right)_{k \geq 1}$ i.i.d. literature.

Proposition 4 (Corollary 5.1, Asmussen (1992)). Assume that the density function of $Z^{+}$satisfies an ODE with constant coefficients described by the polynomial $q_{+}(x)=q_{0}+q_{1} x+\cdots+q_{n} x^{n}$, with non-homogeneous boundary conditions, and denote by $M_{Z^{-}}(s)$ the moment generating function of $Z^{-}$. Define $r_{+}(s)$ through

$$
M_{Z^{+}}(-s)=\frac{r_{+}(s)}{q_{+}(s)},
$$

and assume that for $x_{0}$ with $q_{+}\left(x_{0}\right)=0$ we have $r_{+}\left(x_{0}\right) \neq 0$. Then

$$
\psi(u)=\sum_{i=1}^{n} C_{i} u^{m_{i}} e^{-\lambda_{i} u},
$$

where $C_{i}$, and $\lambda_{i} \in \mathbb{C}$, are the solutions of the Lundberg equation

$$
M_{Z^{-}}(s)=\frac{q_{+}(-s)-p r_{+}(-s)}{(1-p) q_{+}(-s)} \quad \text { or equivalently } \quad M_{Z}(s)=1 .
$$

Further, $m_{i} \in\{0, \ldots n\}$ is the multiplicity of the root $\lambda_{i}$ minus 1 . 
Thus, for the slightly less general case, namely when $Z$ itself has a rational moment generating function, one has the following result (see e.g page 270 in Asmussen and Albrecher (2010) $)$.

Proposition 5. Assume that the moment generating function of $Z$ is rational and

$$
M_{Z}(-s)=\frac{r(s)}{q(s)}
$$

and also assume that for $x_{0}$ with $q\left(x_{0}\right)=0$ we have $r\left(x_{0}\right) \neq 0$. Then the probability of ruin is a solution of the $O D E$

$$
\left(q\left(\frac{d}{d u}\right)-r\left(\frac{d}{d u}\right)\right) \psi(u)=0
$$

Namely it has form (12), where $\lambda_{i}$ are the solutions of the characteristic equation

$$
q(s)-r(s)=0,
$$

with multiplicity $m_{i}$ plus 1 . This is equivalent to saying that $\psi$ has a rational Laplace transform,

$$
\hat{\psi}(s)=\frac{\left((q-r)_{+}(s)-d q_{+}(s)\right)}{s(q-r)_{+}(s)}
$$

where $(q-r)_{+}(s)$ is the polynomial that has as only roots the positive roots of the characteristic equation (14) and $d=(q-r)_{+}(0) / q_{+}(0)$.

Remark 3. After partial fractions decomposition and inversion of the Laplace transform, one obtains the explicit form of the probability of ruin this time down to constants, not only up to constants as in the theorem.

As examples of the $Z$-rational-mgf scenario, one can revisit all the models considered by Ambagaspitiva (2009) and derive exact expressions for the ruin probability. For exemplification, we consider in the following the Kibble and Moran's bivariate Gamma joint density for $\tau$ and $X$, defined through

$$
M_{Z}(s)=\mathbb{E}\left(e^{s Z}\right)=\mathbb{E}\left(e^{-c \tau+X}\right)=M_{\tau, X}(s)=\frac{1}{\left(\left(1+\frac{c s}{\beta_{1}}\right)\left(1-\frac{s}{\beta_{2}}\right)+\rho \frac{c s^{2}}{\beta_{1} \beta_{2}}\right)^{m}},
$$

where $\rho \geq 0$ is the correlation coefficient between $\tau$ and $X$.

Example 1 (Kibble and Moran's bivariate Gamma joint density). According to Proposition 5 one needs to solve first the Lundberg equation

$$
M_{Z}(s)=1 \quad \text { or equivalentely }\left(\left(1+\frac{c s}{\beta_{1}}\right)\left(1-\frac{s}{\beta_{2}}\right)+\rho \frac{c s^{2}}{\beta_{1} \beta_{2}}\right)^{m}=1 .
$$

Assuming that $c \beta_{2}>\beta_{1}$, we get the roots (compare Ambaqaspitiva (2009))

$$
\lambda_{ \pm}^{i}=\frac{c \beta_{2}-\beta_{1} \pm \sqrt{\left(c \beta_{2}-\beta_{1}\right)^{2}+4 c(1-\rho) \beta_{1} \beta_{2}\left(1-e^{4 \pi k / m}\right)}}{2 c(1-\rho)},
$$

where $\operatorname{Re}\left(\lambda_{+}^{i}\right)>0$ and $\operatorname{Re}\left(\lambda_{-}^{i}\right) \leq 0$. Hence $\psi$ satisfies the ODE

$$
\left(\left(\left(1-\frac{c}{\beta_{1}} \frac{d}{d u}\right)\left(1+\frac{1}{\beta_{2}} \frac{d}{d u}\right)+\rho \frac{c^{2}}{\beta_{1} \beta_{2}}\left(\frac{d}{d u}\right)^{2}\right)^{m}-1\right) \psi(u)=0
$$


with solution

$$
\psi(u)=\sum_{i=1}^{m} c_{i} e^{-\lambda_{+}^{i} u} .
$$

Further, note that for $i=1, \ldots, m-1$,

$$
c_{i}=\left.\frac{s+\lambda_{i}}{s} \frac{(q-r)_{+}(s)-d q_{+}(s)}{(q-r)_{+}(s)}\right|_{s=-\lambda_{i}} .
$$

\section{5. $\left(Z_{k}\right)_{k>1}$ dependent with exponential steps/densities}

In this section we return to the dependent case endowed with a Markov chain structure and provide an example for the results derived. We are again in the set-up of $g^{i}(x), i=1, \ldots 4$ being non-constant functions of the initial step $Z_{0}=x$. Let

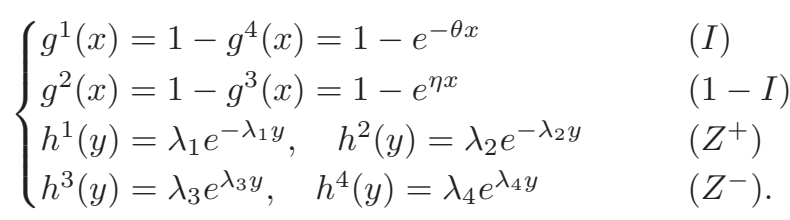

More precisely we will further assume that $\lambda_{1}=\lambda_{2}$ and $\lambda_{3}=\lambda_{4}$. The interpretation of such a model is the following: The size of the jumps are exponentially distributed, having different parameters for positive and negative jumps. Thus, the functions $h^{1}, h^{2}$ and respectively $h^{3}, h^{4}$ play the same role as the densities of $Z^{+}$and $Z^{-}$of (2) in the independent random-walk case of Asmussen (1992). The parameters $\theta$ or $\eta$ control the probability of going up or down in the next step of the random walk. Functions $g^{1}, g^{4}$ (reps. $g^{2}, g^{3}$ ) correspond to $1-I, I$ for $x>0$ (respectively $x<0$ ) as in the decomposition (2). If $\theta$ is large, then it is more likely that a positive jump is followed by another positive jump. On the contrary, if $\eta$ is close to zero then it is more likely that a negative jump is followed by another negative jump (see Figure 1 compare the graph to paths when sampled from the invariant distribution in Figure 21). Since the lucky events are when the random walk goes to $-\infty$, choosing a large $\theta$ one would emphasize the bad tendencies. On the other hand, choosing a $\eta$ close to zero would emphasize the good tendencies. Also, remark that choosing a small $\theta$ would lead to some counter-cyclical, tamed behavior (see Figure 4 the invariant distribution is the same as the one of the model in Figure 1).

Next we give a numerical example. We use the parameters $\lambda_{1}=\lambda_{2}=1$, $\lambda_{3}=\lambda_{4}=4 / 5, \theta=2$ and $\eta=0.4$ (see Figure 3 for a plot of some paths of this process). Using Lemma 4 from the Appendix we derive the density of the invariant distribution,

$$
f_{Z}(x)= \begin{cases}\frac{1}{2} e^{-x} & x \geq 0 \\ \frac{1}{2} \frac{4}{5} e^{\frac{4}{5} x} & x<0 .\end{cases}
$$

With plots 1 and 4 we emphasize the difference in the behavior of the paths when $\theta$ and $\eta$ have different values, even though they have the same invariant 
distribution. Since $\psi^{1}(u)=\psi^{2}(u)$ and $\psi^{3}(u)=\psi^{4}(u)$, to calculate $\psi^{i}(u)$ one needs to solve the system of ODEs

$$
A\left(\begin{array}{l}
\psi^{1} \\
\psi^{4}
\end{array}\right)=\left(\begin{array}{l}
0 \\
0
\end{array}\right) .
$$

In this case, the matrix $A$ is

$$
A=\left(\begin{array}{cc}
\frac{\left(r^{1,1}-q^{1,1}\right) q^{4,1}}{g c d\left(q^{1,1}, q^{4,1}\right)} & \frac{q^{1,1} r^{4,1}}{g c d\left(q^{1,1}, q^{4,1}\right)} \\
\frac{r^{2,3} q^{3,3}}{\operatorname{gcd}\left(q^{2,3}, q^{3,3}\right)} & \frac{\left(r^{3,3}-q^{3,3}\right) q^{2,3}}{\operatorname{gcd}\left(q^{2,3}, q^{3,3}\right)}
\end{array}\right),
$$

with the determinant

$$
\operatorname{det}(A)=s\left(s^{3}+\frac{14 s^{2}}{5}-\frac{87 s}{25}-\frac{6}{25}\right) .
$$

Given the infinity condition, one needs to consider only the negative roots of the polynomial $\operatorname{det}(A)=0$. Thus, the solution of the system of ODEs has the form

$$
\psi^{i}(u)=c_{2}^{i} e^{-3.7185 u}+c_{2}^{i} e^{-0.0655856 u},
$$

which once plugged back into Equation (10) leads to

$$
\begin{aligned}
& \psi^{1}(u)=\psi^{2}(u)=0.0488089 e^{-3.7185 u}+0.951191 e^{-0.0655856 u} \\
& \psi^{3}(u)=\psi^{4}(u)=0.000839295 e^{-3.7185 u}+0.75528 e^{-0.0655856 u}
\end{aligned}
$$

and thus

$$
\psi(u, x)= \begin{cases}\left(1-e^{-2 x}\right) \psi^{1}(u)+e^{-2 x} \psi^{3}(u) & x>0 \\ \left(1-e^{\frac{4}{10} x}\right) \psi^{1}(u)+e^{-\frac{4}{10} x} \psi^{3}(u) & x<0 .\end{cases}
$$

Further, if we assume that $Z_{0}$ is distributed according to the invariant distribution, then

$$
\psi(u)=0.0248241 e^{-3.7185 u}+0.853236 e^{-0.0655856 u} .
$$

We compare this to the ruin probability of a process with iid increments, which follows the same invariant distribution,

$$
\psi_{\text {indep }}(u)=\frac{9}{10} e^{-\frac{u}{10}} .
$$

Numerical values of these ruin probabilities are plotted in Figures 5 and 6 One can clearly see that, in this case, dependence causes an increase of the risk of ruin.

\section{Conclusions}

One of the fundamental problems in random walk theory is the computation of the ladder height distributions. More specifically, for a random walk

$$
W_{n}=\sum_{k=1}^{n} Z_{k}
$$




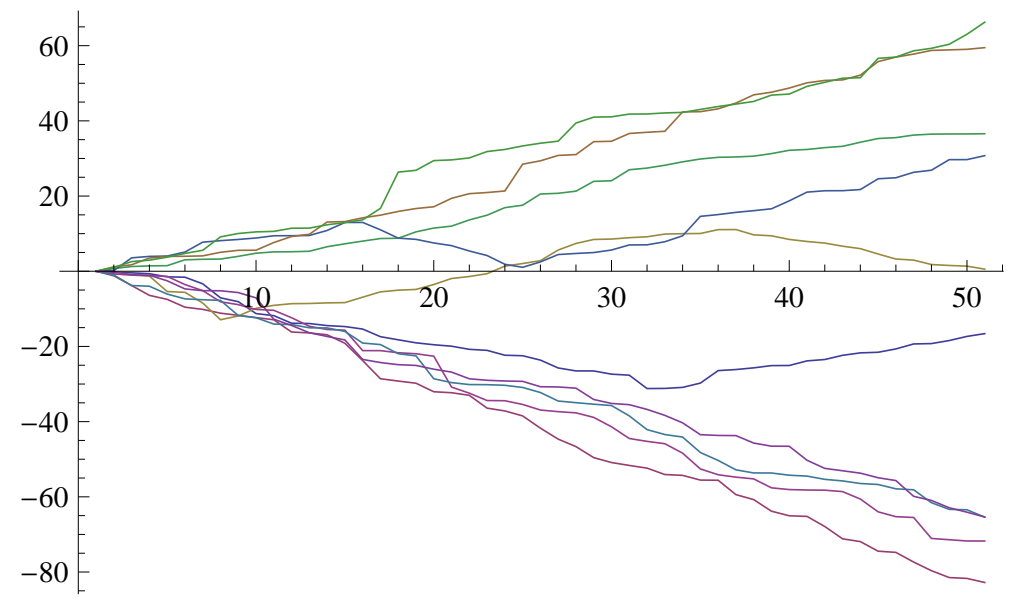

Figure 1: Paths of the risk process when $\lambda_{1}=\lambda_{2}=1, \lambda_{3}=\lambda_{4}=4 / 5, \theta=100$ and $\eta=0.008$

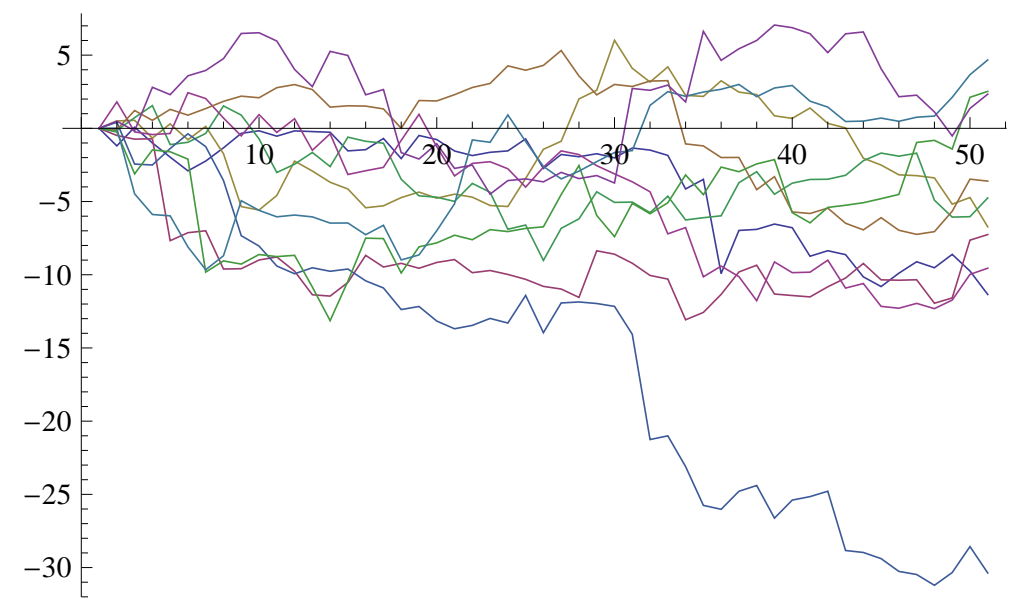

Figure 2: Paths of the risk process when the claims are iid distributed with the invariant distribution of a process with $\lambda_{1}=\lambda_{2}=1, \lambda_{3}=\lambda_{4}=4 / 5, \theta=100$ and $\eta=0.008$ 


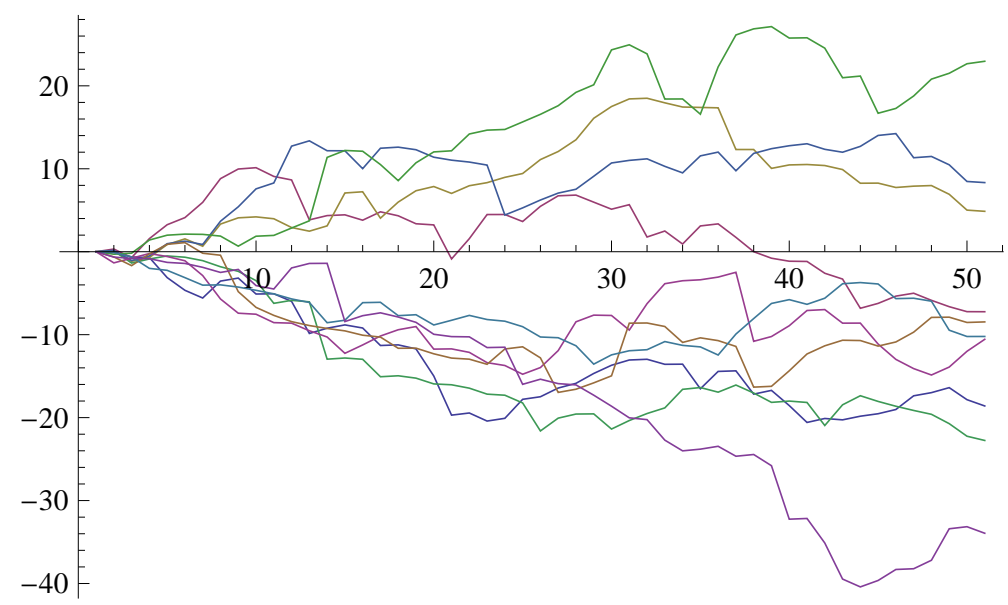

Figure 3: Paths of the risk process when $\lambda_{1}=\lambda_{2}=1, \lambda_{3}=\lambda_{4}=4 / 5, \theta=2$ and $\eta=0.4$

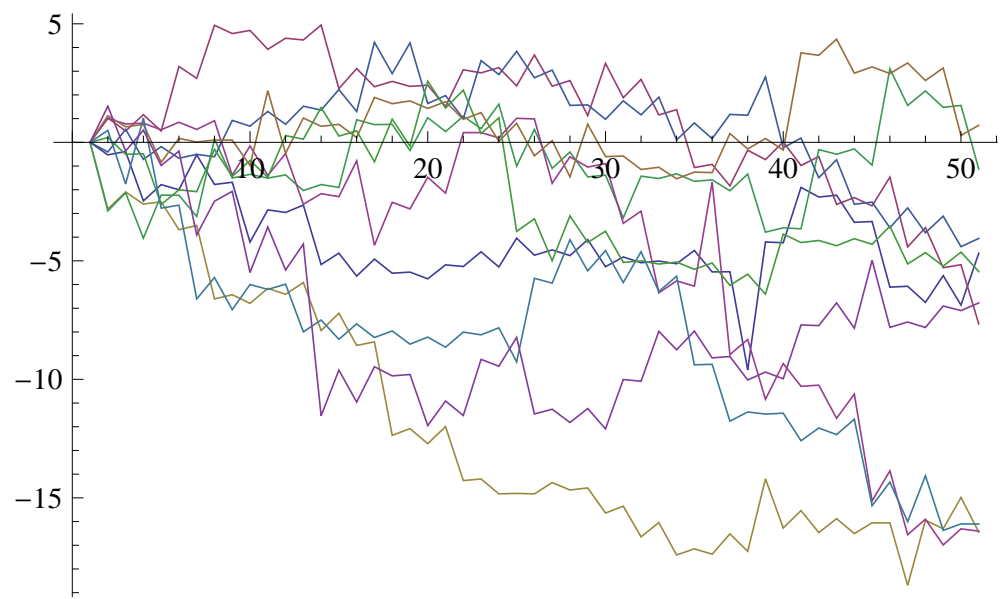

Figure 4: Paths of the risk process when $\lambda_{1}=\lambda_{2}=1, \lambda_{3}=\lambda_{4}=4 / 5, \theta=100^{-1}$ and $\eta=80$ 


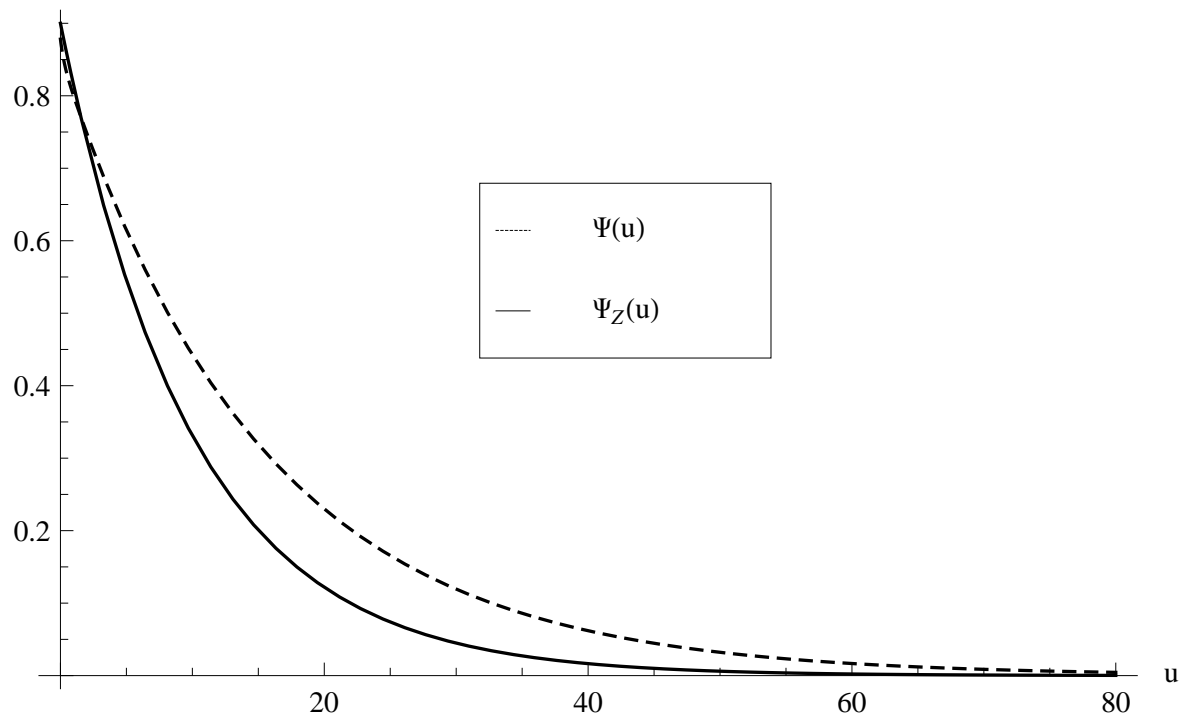

Figure 5: Comparison of the ruin probability (absolute values) of the Markov chain with $\lambda_{1}=\lambda_{2}=1, \lambda_{3}=\lambda_{4}=4 / 5, \theta=2$ and $\eta=0.4$, and the corresponding process of independent $Z$ with the invariant distribution.

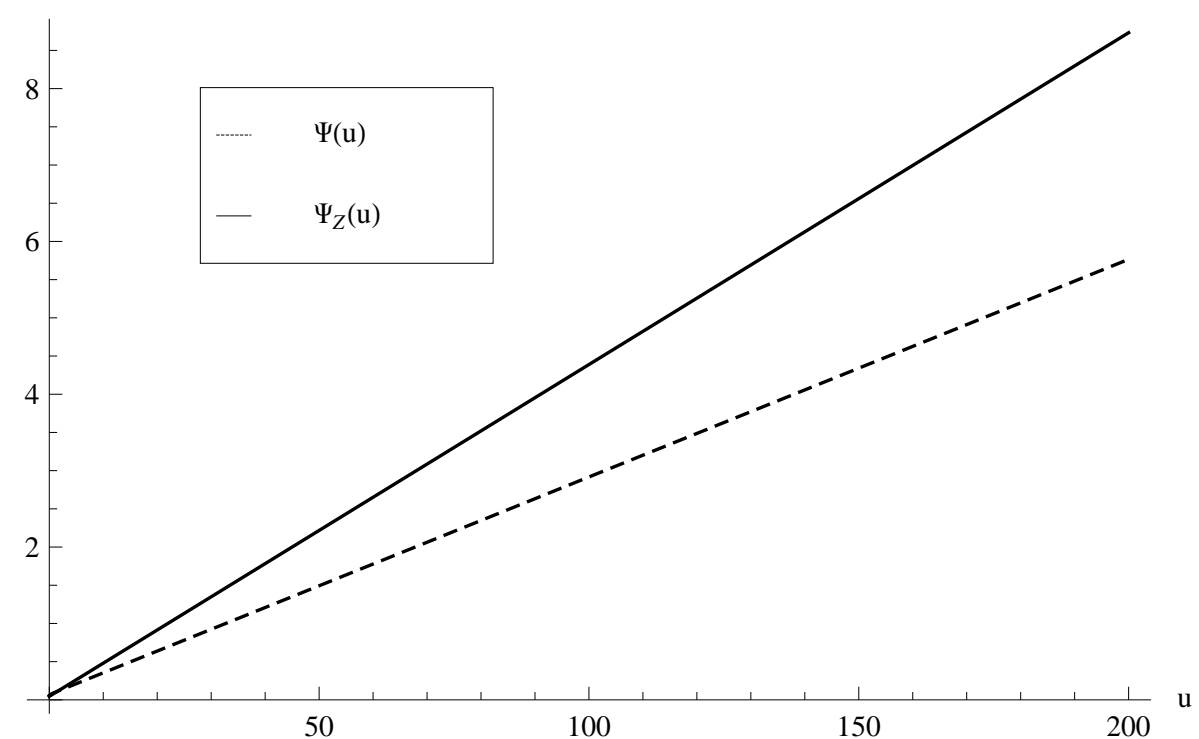

Figure 6: Comparison of the ruin probability ( $-\log _{10}$ of the values) of the Markov chain with $\lambda_{1}=\lambda_{2}=1, \lambda_{3}=\lambda_{4}=4 / 5, \theta=2$ and $\eta=0.4$, and the corresponding process of independent $Z$ with the invariant distribution. 
with increments having the distribution $F_{Z}$ and ladder epochs defined as $\tau_{-}=$ $\inf \left\{n \geq 1 \mid W_{n} \geq 0\right\}$ respectively $\tau_{+}=\inf \left\{n \geq 1 \mid W_{n}>0\right\}$, the ladder height distributions are $G_{-}(u)=\mathbb{P}\left(Z_{\tau_{-}} \leq u\right)$ and $G_{+}(u)=\mathbb{P}\left(Z_{\tau_{+}} \leq u\right)$. These quantities are relevant in sequential, queuing and risk theory. Results have been derived for increments having a structure $Z=p Z_{1}+(1-p) Z_{2}$ (Feller, 1971) or a difference structure $Z=\tau-X$ (Asmussen, 1992).

Focusing on a risk theory model, one could analyze the probability of ruin in this setting. In this paper we base our analysis on the real-valued random variable $Z_{k}=-c \tau_{k}+X_{k}$ describing the difference between the inter-arrival time $\tau_{k}$ and its consecutive claim size $X_{k}$. In the classical model, $\tau$ and $X$ are assumed to be independent. If regardless of the dependence structure of $\tau$ and $X, Z_{k}$ are independent, we are in the random walk setting. A natural condition for $Z$ in order to be able to derive explicit forms of the ruin probability is that its $\mathrm{mgf}$ is rational. Or, the weaker/more general version of it, that only the $\mathrm{mgf}$ of $Z^{+}=\{Z \mid Z>0\}$ is rational.

Once we step away from the independence of $Z_{k}$, there are a multitude of dependence structures possible. We introduced a Markov chain dependence which still permits the exact calculation of the probability of ruin. A motivation for such a model, besides the mathematical amenability, consists in the fact that one can implement a counter-cyclic behavior of the sample paths. Although a step away from the classical random walk setting, the model and the method can be presented as an immediate alteration/extension of it.

Acknowledgments. This work has been partially supported by the French Research National Agency (ANR) under the reference ANR-08-BLAN-0314-01 and the Swiss National Science Foundation Project 200021-124635/1.

\section{Lemma 1.}

1. If the joint moment generating function $M_{\tau, X}(t, s)$ of the vector $(\tau, X)$ is rational, then the moment generating functions of $Z^{+}$and $Z^{-}, M_{Z^{+}}$and respectively $M_{Z^{-}}$are also rational.

2. If $M_{Z^{+}}$and $M_{Z^{-}}$are rational, then there exists a vector $(\tau, X)$ such that $M_{\tau, X}(t, s)$ is rational.

Proof. Ad 1 Since $M_{\tau, X}(t, s)$ is rational, the density $F_{Z}$ of $Z=X-c \tau=$ $I Z^{+}+(1-I) Z^{-}$has a rational Fourier transform, implying

$$
f_{Z}(x)=\sum_{i=1}^{n} c_{i} x^{m_{i}} e^{-\lambda_{i} x} 1_{\left\{\operatorname{sign}\left(\operatorname{Re}\left(\lambda_{i}\right)\right)[0, \infty]\right\}}(x) .
$$

Now 1 follows from

$$
p f_{Z^{+}}(x)=\sum_{i=1, \operatorname{Re}\left(\lambda_{i}\right)>0}^{n} c_{i} x^{m_{i}} e^{-\lambda_{i} x} 1_{\{(0, \infty)\}}(x)
$$

and

$$
(1-p) f_{Z^{-}}(x)=\sum_{i=1, \operatorname{Re}\left(\lambda_{i}\right)<0}^{n} c_{i} x^{m_{i}} e^{-\lambda_{i} x} 1_{\{(-\infty, 0)\}}(x) .
$$


Ad 2] We have

$$
M_{Z}(s)=p M_{Z^{+}}(s)+(1-p) M_{Z^{-}}(s)=\frac{p}{1-s+s} M_{Z^{+}}(s)+\frac{1-p}{1-s+s} M_{Z^{-}}(s) .
$$

We use

$$
M_{\tau, X}(t, s)=p \frac{1}{1-t-s} M_{Z^{+}}(s)+(1-p) \frac{1}{1-t-s} M_{Z^{-}}(-t) .
$$

To show that this is the moment generating function of a random vector, let $I$ be $\operatorname{Bernoulli}(p)$ and $Y$ be $\operatorname{Exp}(1)$. Then the random vector

$$
(\tau, X):=\mathbb{P}\left(Y, Y+Z^{+}\right)+(1-P)\left(Y-Z^{-}, Y\right)
$$

has $M_{\tau, X}(t, s)$ as moment generating function.

Lemma 2. Let $g(y)$ and $\psi(y)$ be two functions that are sufficiently often differentiable and bounded for $y>0$. Assume that there exists a polynomial $q(x)=q_{0}+q_{1} x+\cdots+q_{n} x^{n}$ with

$$
q\left(\frac{d}{d y}\right) g(y)=0, \quad y>0 .
$$

Further define the polynomial

$$
r(x)=\sum_{l=0}^{n-1} \sum_{i=l+1}^{n} q_{i} g^{(i-l-1)}(0) x^{l} .
$$

Then the Laplace transform of $g$

$$
\int_{0}^{\infty} e^{-s y} g(y) d y=\frac{r(s)}{q(s)}
$$

and

$$
q\left(\frac{d}{d u}\right) \int_{0}^{u} \psi(u-y) g(y) d y=r\left(\frac{d}{d u}\right) \psi(u) .
$$

Proof. Note that for $s>0$, using integration be parts

$$
\int_{0}^{\infty} e^{-s y} g(y) d y=\sum_{k=1}^{l} \frac{1}{s^{k}} g^{(k-1)}(0)+\int_{0}^{\infty} \frac{1}{s^{l}} e^{-s x} g^{(l)}(x) d x .
$$

Multiplying with $q(s)$ leads to

$$
\begin{aligned}
q(s) \int_{0}^{\infty} e^{-s y} g(y) d y & =\sum_{l=0}^{n}\left(q_{l} \sum_{k=1}^{l} s^{l-k} g^{(k-1)}(0)+\int_{0}^{\infty} e^{-s x} q_{l} g^{(l)}(x) d x\right) \\
& =\sum_{k=0}^{n-1} \sum_{l=k+1}^{n} q_{l} g^{(l-k-1)}(0) s^{k}+\int_{0}^{\infty} e^{-s x} q\left(\frac{d}{d x}\right) g(x) d x \\
& =r(s) .
\end{aligned}
$$


For the second part of the lemma note that by induction

$$
\begin{aligned}
\left(\frac{d}{d u}\right)^{n} \int_{0}^{u} \psi(u-y) g(y) & d y=\left(\frac{d}{d u}\right)^{n} \int_{0}^{u} \psi(y) g(u-y) d y \\
= & \sum_{k=0}^{n-1} \psi^{(k)}(u) g^{(n-k-1)}(0)+\int_{0}^{u} \psi(y) g^{(n)}(u-y) d y
\end{aligned}
$$

It follows

$$
\begin{aligned}
q\left(\frac{d}{d u}\right) \int_{0}^{u} \psi(u-y) g(y) d y & =\sum_{l=0}^{n} q_{l} \sum_{k=0}^{l-1} \psi^{(k)}(u) g^{(n-k-1)}(0) \\
& +\int_{0}^{u} \psi(y) q\left(\frac{d}{d u}\right) g(u-y) d y=r\left(\frac{d}{d u}\right) \psi(u) .
\end{aligned}
$$

Lemma 3. Let $g(y)$ and $\psi(y)$ be two functions that are sufficiently often differentiable, where $g(y)$ is bounded for $y<0$ and $\psi(y)$ is bounded for $y>0$. Assume that there exists a polynomial $q(x)=q_{0}+q_{1} x+\cdots+q_{n} x^{n}$ with

$$
q\left(\frac{d}{d y}\right) g(y)=0, \quad y<0 .
$$

Further define the polynomial

$$
r(x)=-\sum_{l=0}^{n-1} \sum_{i=l+1}^{n} q_{i} g^{(i-l-1)}(0) x^{l} .
$$

Then

$$
\int_{-\infty}^{0} e^{-s y} g(y) d y=\frac{r(s)}{q(s)}
$$

and

$$
q\left(\frac{d}{d u}\right) \int_{-\infty}^{0} \psi(u-y) g(y) d y=r\left(\frac{d}{d u}\right) \psi(u) .
$$

Proof. At first note that by partial integration

$$
\int_{-\infty}^{0} e^{-s y} g(y) d y=-\sum_{k=1}^{l} \frac{1}{s^{k}} g^{(k-1)}(0)+\int_{-\infty}^{0} \frac{1}{s^{l}} e^{-s x} g^{(l)}(x) d x .
$$

Multiplying with $q(s)$ leads to

$$
\begin{aligned}
q(s) \int_{-\infty}^{0} e^{-s y} g(y) d y & =-\sum_{l=0}^{n}\left(q_{l} \sum_{k=1}^{l} s^{l-k} g^{(k-1)}(0)-\int_{-\infty}^{0} e^{-s x} q_{l} g^{(l)}(x) d x\right) \\
& =-\sum_{k=0}^{n-1} \sum_{l=k+1}^{n} q_{l} g^{(l-k-1)}(0) s^{k}+\int_{0}^{\infty} e^{-s x} q\left(\frac{d}{d x}\right) g(x) d x \\
& =r(s) .
\end{aligned}
$$


For the second part of the lemma note that by induction

$$
\begin{aligned}
\left(\frac{d}{d u}\right)^{n} \int_{-\infty}^{0} \psi(u-y) g(y) d y & =\left(\frac{d}{d u}\right)^{n} \int_{u}^{\infty} \psi(y) g(u-y) d y \\
& =-\sum_{k=0}^{n-1} \psi^{(k)}(u) g^{(n-k-1)}(0)+\int_{u}^{\infty} \psi(y) g^{(n)}(u-y) d y
\end{aligned}
$$

It follows

$$
\begin{aligned}
& q\left(\frac{d}{d u}\right) \int_{u}^{\infty} \psi(u-y) g(y) d y=-\sum_{l=0}^{n} q_{l} \sum_{k=0}^{l-1} \psi^{(k)}(u) g^{(n-k-1)}(0) \\
&+\int_{u}^{\infty} \psi(y) q\left(\frac{d}{d u}\right) g(u-y) d y=r\left(\frac{d}{d u}\right) \psi(u)
\end{aligned}
$$

Lemma 4. Let $Z$ be a Markov chain with transition density satisfying the Assumption [1] Denote with

$$
K^{l, m}=\left\{\begin{array}{ll}
\int_{0}^{\infty} g^{l}(x) h^{m}(x) d x, & \text { if } l \in\{1,4\}, m \in\{1,2\} \\
\int_{-\infty}^{0} g^{l}(x) h^{m}(x) d x, & \text { if } l \in\{2,3\}, m \in\{3,4\}
\end{array} .\right.
$$

Then the invariant distribution has the density

$$
\begin{aligned}
& f_{Z}(y)=\frac{K^{1,2} K^{2,3} h^{1}(y)+K^{2,3} K^{4,1} h^{2}(y)}{K^{1,2} K^{2,3}+2 K^{2,3} K^{4,1}+K^{3,4} K^{4,1}}, \quad y>0, \\
& f_{Z}(y)=\frac{K^{3,4} K^{4,1} h^{3}(y)+K^{2,3} K^{4,1} h^{4}(y)}{K^{1,2} K^{2,3}+2 K^{2,3} K^{4,1}+K^{3,4} K^{4,1}}, \quad y<0,
\end{aligned}
$$

Proof. The invariant distribution is defined through

$$
f_{Z}(y)=\int_{0}^{\infty} p(x, y) f_{Z}(x) d x
$$

We can split this equation into two equations, for $y>0$ and $y<0$,

$$
\begin{aligned}
& f_{Z}(y)=h^{1}(y) \int_{0}^{\infty} g^{1}(x) f_{Z}(x) d x+h^{2}(y) \int_{-\infty}^{0} g^{2}(x) f_{Z}(x) d x, \quad y>0 \\
& f_{Z}(y)=h^{4}(y) \int_{0}^{\infty} g^{4}(x) f_{Z}(x) d x+h^{3}(y) \int_{-\infty}^{0} g^{3}(x) f_{Z}(x) d x, \quad y<0 .
\end{aligned}
$$

It follows that the invariant distribution can be written as

$$
\begin{array}{ll}
f_{Z}(y)=c^{1} h^{1}(y)+c^{2} h^{2}(y), & y>0, \\
f_{Z}(y)=c^{3} h^{3}(y)+c^{4} h^{4}(y), & y<0,
\end{array}
$$

and we are left with determining the constants $c^{l}$, which are the solution of the system of linear equations

$$
\left\{\begin{array}{l}
c^{1}=K^{1,1} c^{1}+K^{1,2} c^{2} \\
c^{2}=K^{2,3} c^{4}+K^{2,4} c^{4} \\
c^{3}=K^{3,3} c^{4}+K^{3,4} c^{4} \\
c^{4}=K^{4,1} c^{1}+K^{4,2} c^{2} .
\end{array}\right.
$$


In our matrix notation

$$
\left(\begin{array}{cccc}
K^{1,1}-1 & K^{1,2} & 0 & 0 \\
0 & -1 & K^{2,3} & K^{2,4} \\
0 & 0 & K^{3,3}-1 & K^{3,4} \\
K^{4,1} & K^{4,2} & 0 & -1
\end{array}\right)\left(\begin{array}{l}
c^{1} \\
c^{2} \\
c^{3} \\
c^{4}
\end{array}\right)=\left(\begin{array}{l}
0 \\
0 \\
0 \\
0
\end{array}\right)
$$

Using the fact that $\int h^{i}(x) d x=1$ and $1-g^{i}(x)=g^{5-i}(x)$ one can rewrite the matrix in (.1) as

$$
\left(\begin{array}{cccc}
-K^{4,1} & 1-K^{4,2} & 0 & 0 \\
0 & -1 & K^{2,3} & K^{2,4} \\
0 & 0 & -K^{2,3} & 1-K^{2,4} \\
K^{4,1} & K^{4,2} & 0 & -1
\end{array}\right)
$$

Moreover, by adding all other rows to the last one, it becomes

$$
\left(\begin{array}{cccc}
-K^{4,1} & 1-K^{4,2} & 0 & 0 \\
0 & -1 & K^{2,3} & K^{2,4} \\
0 & 0 & -K^{2,3} & 1-K^{2,4} \\
0 & 0 & 0 & 0
\end{array}\right)\left(\begin{array}{l}
c^{1} \\
c^{2} \\
c^{3} \\
c^{4}
\end{array}\right)=\left(\begin{array}{l}
0 \\
0 \\
0 \\
0
\end{array}\right)
$$

It follows that

$$
\begin{aligned}
c^{1} & =t K^{1,2}\left(\frac{K^{3,4}+K^{2,4}}{K^{4,1}}\right)=t \frac{K^{1,2}}{K^{4,1}} \\
c^{2} & =t\left(K^{3,4}+K^{2,4}\right)=t \\
c^{3} & =t \frac{K^{3,4}}{K^{2,3}} \\
c^{4} & =t .
\end{aligned}
$$

Since $f_{Z}(x)$ is a density function, $t^{-1}=\frac{K^{1,2}}{K^{4,1}}+\frac{K^{3,4}}{K^{2,3}}+2$.

Remark 4. In the general case we have that $\int h^{i}(x) d x=1$ and $1-\sum_{j} g_{j}^{i}(x)=$ $\sum_{j} g_{j}^{5-i}(x)$ and hence for fixed $j$ and $m$,

$$
\sum_{i} K_{i, j}^{l, m}+K_{i, j}^{5-l, m}=1
$$

$\left(K_{i, j}^{l, m}\right.$ is defined as $K^{l, m}$ by replacing $h^{m}$ by $h_{j}^{m}$ and $g^{l}$ by $\left.g_{i}^{l}\right)$. If we interpret the matrix (.1) as a block matrix where $K^{l, m}$ corresponds to the matrix with elements $K_{i, j}^{l, m}$ and 1 to the identity matrix, then the sum of all rows is 0 . Which means that we get non-trivial candidates for $f_{Z}$.

Proof of Proposition 1. Equation (5) for $\psi(u, x)$ will have two expressions, de- 
pending on the sign of $x$. For $x \in I_{1} \cup I_{4},(x>0)$

$$
\begin{aligned}
\psi(u, x)= & \int_{u}^{\infty} g^{1}(x) h^{1}(y) d y+\int_{-\infty}^{0} \psi(u-y, y) g^{4}(x) h^{4}(y) d y \\
& +\int_{0}^{u} \psi(u-y, y) g^{1}(x) h^{1}(y) d y \\
= & g^{1}(x)\left(\int_{u}^{\infty} h^{1}(y) d y+\int_{0}^{u} \psi(u-y, y) h^{1}(y) d y\right) \\
& +g^{4}(x) \int_{-\infty}^{0} \psi(u-y, y) h^{4}(y) d y \\
= & g^{1}(x) \psi^{1}(u)+g^{4}(x) \psi^{4}(u) .
\end{aligned}
$$

Analogously for $x \in I_{2} \cup I_{3},(x<0)$

$$
\begin{aligned}
\psi(u, x)= & \int_{u}^{\infty} g^{2}(x) h^{2}(y) d y+\int_{-\infty}^{0} \psi(u-y, y) g^{3}(x) h^{3}(y) d y \\
& +\int_{0}^{u} \psi(u-y, y) g^{2}(x) h^{2}(y) d y \\
= & g^{2}(x)\left(\int_{u}^{\infty} h^{2}(y) d y+\int_{0}^{u} \psi(u-y, y) h^{2}(y) d y\right) \\
& +g^{3}(x) \int_{-\infty}^{0} \psi(u-y, y) h^{3}(y) d y \\
= & g^{2}(x) \psi^{2}(u)+g^{3}(x) \psi^{3}(u) .
\end{aligned}
$$

Thus the first part of the theorem follows. The second part of the theorem follows from $\lim _{u \rightarrow \infty} \psi(u, x)=0$ for every $x$.

Proof of Theorem From the fact that $\psi^{i}$ solve a system of ODEs with boundary conditions $\lim _{u \rightarrow \infty} \psi^{i}(u)=0$, we have

$$
\psi^{i}(u)=\sum_{j=1}^{n} \sum_{k=0}^{m} c_{j, k}^{i} u^{k} e^{-\lambda_{j} u},
$$

where $-\lambda_{j}$ are the negative roots of the polynomial

$$
\operatorname{det}\left(\begin{array}{cccc}
\frac{\left(r^{1,1}-q^{1,1}\right) q^{4,1}}{g c d\left(q^{1,1} q^{4,1}\right)} & 0 & 0 & \frac{q^{1,1} r^{4,1}}{g c d\left(q^{1,1}, q^{4,1}\right)} \\
\frac{r^{1,2}, q^{4,2}}{g c d\left(q^{1,2}, q^{4,2}\right)} & -\frac{q^{1,2} q^{4,2}}{g c d\left(q^{1,2, q^{4,2}}\right.} & 0 & \frac{q^{1,2} r^{4,2}}{g c d\left(q^{1,2}, q^{4,2}\right)} \\
0 & \frac{r^{2,3} q^{3,3}}{g c d\left(q^{2,3} q^{3,3}\right)} & \frac{\left(r^{3,3}-q^{3,3}\right) q^{2,3}}{g c d\left(q^{2,3} q^{3,3}\right)} & 0 \\
0 & \frac{r^{2,4} q^{3,4}}{\operatorname{gcd}\left(q^{2,4}, q^{3,4}\right)} & \frac{q^{2,4} r^{3,4}}{\operatorname{gcd}\left(q^{2,4}, q^{3,4}\right)} & -\frac{q^{2,4} q^{3,4}}{\operatorname{gcd}\left(q^{2,4}, q^{3,4}\right)},
\end{array}\right)=0
$$

equivalent to

$$
\begin{aligned}
& 0=\frac{\left(r^{1,1}-q^{1,1}\right)\left(r^{3,3}-q^{3,3}\right) q^{1,2} q^{2,3} q^{2,4} q^{3,4} q^{4,1} q^{4,2}}{g c d\left(q^{1,1}, q^{4,1}\right) \operatorname{gcd}\left(q^{1,2}, q^{4,2}\right) g c d\left(q^{2,3}, q^{3,3}\right) g c d\left(q^{2,4}, q^{3,4}\right)} \\
& +\frac{\left(r^{2,3} r^{3,4} q^{2,4} q^{3,3}-r^{2,4}\left(r^{3,3}-q^{3,3}\right) q^{2,3} q^{3,4}\right)\left(r^{4,2}\left(r^{1,1}-q^{1,1}\right) q^{1,2} q^{4,1}-r^{1,2} r^{4,1} q^{1,1} q^{4,2}\right)}{\operatorname{gcd}\left(q^{1,1}, q^{4,1}\right) \operatorname{gcd}\left(q^{1,2}, q^{4,2}\right) \operatorname{gcd}\left(q^{2,3}, q^{3,3}\right) g c d\left(q^{2,4}, q^{3,4}\right)} .
\end{aligned}
$$


Dividing by

$$
\frac{q^{1,1} q^{1,2} q^{2,3} q^{2,4} q^{3,3} q^{3,4} q^{4,1} q^{4,2}}{\operatorname{gcd}\left(q^{1,1}, q^{4,1}\right) \operatorname{gcd}\left(q^{1,2}, q^{4,2}\right) \operatorname{gcd}\left(q^{2,3}, q^{3,3}\right) \operatorname{gcd}\left(q^{2,4}, q^{3,4}\right)}
$$

leads to the equation

$$
\begin{array}{r}
0=\frac{\left(r^{1,1}-q^{1,1}\right)\left(r^{3,3}-q^{3,3}\right)}{q^{1,1} q^{3,3}}+\frac{r^{2,3} r^{3,4} r^{4,2}\left(r^{1,1}-q^{1,1}\right)}{q^{1,1} q^{2,3} q^{3,4} q^{4,2}}-\frac{r^{1,2} r^{2,3} r^{3,4} r^{4,1}}{q^{1,2} q^{2,3} q^{3,4} q^{4,1}} \\
-\frac{\left(r^{1,1}-q^{1,1}\right)\left(r^{3,3}-q^{3,3}\right) r^{2,4} r^{4,2}}{q^{1,1} q^{2,4} q^{3,3} q^{4,2}}+\frac{\left(r^{3,3}-q^{3,3}\right) r^{1,2} r^{2,4} r^{4,1}}{q^{1,2} q^{2,4} q^{3,3} q^{4,1}}
\end{array}
$$

The claim follows from the definition of $M_{k, m}$.

\section{References}

Hansjörg Albrecher and Jef L. Teugels. Exponential behavior in the presence of dependence in risk theory. J. Appl. Probab., 43(1):257-273, 2006.

Hansjörg Albrecher, Corina Constantinescu, Gottlieb Pirsic, Georg Regensburger, and Markus Rosenkranz. An algebraic operator approach to the analysis of Gerber-Shiu functions. Insurance Math. Econom., 46(1):42-51, 2010 .

Rohana S. Ambagaspitiya. Ultimate ruin probability in the Sparre Andersen model with dependent claim sizes and claim occurrence times. Insurance Math. Econom., 44(3):464-472, 2009.

Søren Asmussen. Phase-type representations in random walk and queueing problems. Ann. Probab., 20(2):772-789, 1992.

Søren Asmussen and Hansjörg Albrecher. Ruin probabilities. Advanced Series on Statistical Science \& Applied Probability. World Scientific Publishing Co. Inc., River Edge, NJ, 2010.

Andrei L. Badescu, Eric C. K. Cheung, and David Landriault. Dependent risk models with bivariate phase-type distributions. J. Appl. Probab., 46(1):113131,2009 .

Mogens Bladt and Bo Friis Nielsen. Multivariate matrix-exponential distributions. Stochastic Models, 26:1-26, 2010.

Bruno de Finetti. Su un'impostazione alternativa dell teoria collecttiva del rischio. In Trans. XVth Internat. Congress Actuaries, 2:433-443, 1957.

William Feller. An introduction to probability theory and its applications. Vol. II. Second edition. John Wiley \& Sons Inc., New York, 1971. 